HNO 2012 $\cdot 60: 291-292$

DOI 10.1007/s00106-012-2507-0

Online publiziert: 11. April 2012

(c) Springer-Verlag 2012

N. Stasche

HNO-Klinik, Westpfalz-Klinikum Kaiserslautern

\title{
Die Bedeutung der Kopf-Hals-Chirurgie für die Hals-Nasen-Ohren-Heilkunde
}

\author{
83. Jahresversammlung der Deutschen \\ Gesellschaft für Hals-Nasen-Ohren-Heilkunde, \\ Kopf- und Hals-Chirurgie in Mainz, \\ 16.-20. Mai 2012
}

Die 83. Jahresversammlung der Deutschen Gesellschaft für Hals-Nasen-Ohren-Heilkunde, Kopf- und Hals-Chirurgie findet nach 43 Jahren vom 16. bis 20. Mai 2012 zum zweiten Mal in Mainz statt. Im Jahre 1969 war unsere Fachgesellschaft schon einmal mit ihrer Jahresversammlung zu Gast in der Landeshauptstadt von Rheinland-Pfalz. Das unter der Präsidentschaft von Horst Wullstein gestaltete wissenschaftliche Programm beinhaltete damals Referate zum Thema Vestibularisforschung und stellte mit den Hauptvorträgen von W.F. House, U. Fisch und M.G. Yasargil wichtige Weichen für die Entwicklung der Schädelbasischirurgie in der HNO.

\section{Motto: Grenzgebiete}

Die diesjährige Jahresversammlung steht unter dem Motto „Grenzgebiete der Hals-Nasen-Ohrenheilkunde“, welches die Vielseitigkeit und Innovationsfähigkeit unseres Fachgebiets in den Überschneidungen mit den Nachbardisziplinen zum Ausdruck bringen soll. Die vorgelegten neun Referate geben einen aktuellen, evidenzbasierten und interdisziplinären Überblick über die Pathogenese, die Diagnostik und die Therapie von Kopf-Hals-Tumoren. Das Referatethe- ma eignet sich in ganz besonderem Maße dazu, die Bedeutung der klinischen Forschung als Grenzüberschreitung und Beeinflussung der beteiligten Fachdisziplinen sowie die Bedeutung der translationalen Forschung für unser Fachgebiet darzustellen. Vor dem Hintergrund der zahlreichen Berührungspunkte mit benachbarten Fachgebieten werden wir u. a. Themen der interventionellen Endoskopie des oberen Aerodigestivtrakts, der Schluckstörungen, des Schwindels, neuer Evidenzen bei der Therapie des Hörsturzes und der schlafmedizinischen Aspekte des Obesitas-HypoventilationsSyndroms diskutieren. Der Tag der Praxis steht ganz im Zeichen der Allergologie, und beim Pflegetag werden u. a. Konzepte des modernen Wundmanagements vorgestellt.

\section{Kopf-Hals-Chirurgie als fester Bestandteil der HNO}

Seit den ersten Jahresversammlungen unserer Fachgesellschaft ist in einzelnen Vorträgen die Behandlung von KopfHals-Tumoren mehrfach thematisiert worden. Erstmals 1932 in Bad Ems referierte der Hamburger Strahlentherapeut Gottfried Holthusen über die „Strahlentherapie in der Otorhinolaryngologie“.
Lange Jahre wurde die große Tumorchirurgie noch außerhalb des HNO-Fachgebiets betrieben. Erst 1951 auf der Jahresversammlung in Hamburg unter der Präsidentschaft von Otto Steurer beschrieb Johannes Zange aus Jena in seinem Referat die Grundlagen der „Chirurgischen und Strahlentherapie der bösartigen Geschwülste des Kehlkopfes und Kehlkopfrachens". Dieses Referat legte die Grundlage für die bis heute in Deutschland gelebte Einheit von Hals-Nasen-OhrenHeilkunde und Kopf-Hals-Chirurgie, welche dann 1968 mit der Umbenennung unserer Fachgesellschaft in „Deutsche Gesellschaft für Hals-Nasen-Ohren-Heilkunde, Kopf- und Hals-Chirurgie" seinen Ausdruck fand. In der zweiten Hälfte des 20. Jahrhunderts folgte die Blütezeit der radikalen Tumorchirurgie in der HNO mit einer kontinuierlichen Erweiterung der Indikationen auch für ausgedehnte Kopf-Hals-Tumoren. Dies war durch die sich immer weiter entwickelnden Rekonstruktionstechniken mit lokalen Lappenplastiken bis hin zur Einführung mikrovaskulär anastomosierter Transplantate möglich. Trotz dieser Fortschritte in der rekonstruktiven Chirurgie ließ sich die Prognose bei fortgeschrittenen Tumorstadien nicht wesentlich verbessern. Es folgte die rasante Entwick- 
lung der transoralen, funktionserhaltenden Laser-Mikrochirurgie, welche sich bis heute mit der TORS (,transoral robotic surgery") fortsetzt. Mit exzellenten onkologischen und funktionellen Ergebnissen, zunächst für umschriebene Tumorstadien am Kehlkopf entwickelt, spielt die transorale Laser-Mikrochirurgie eine immer größere Rolle auch bei der operativen Behandlung von fortgeschrittenen Tumorstadien.

\section{Rolle der primären Chirurgie bei Kopf-Hals-Tumoren}

Die Operabilität eines Kopf-Hals-Tumors stellt die zentrale Frage bei der Therapieentscheidung und der Abgrenzung der Indikationsstellung für nichtoperative Therapieoptionen dar. Durch die Fortschritte auf dem Gebiet der Tumorbiologie und genetischen Grundlagenforschung ist es zu einer deutlichen Zunahme von nichtoperativen Behandlungsprotokollen gekommen. Die Therapieentscheidungen werden zunehmend von Hämatoonkologen und Radioonkologen getroffen, welche die interdisziplinären Tumorboards dominieren. GroBe prospektive Multizenterstudien zur Therapie von Kopf-Hals-Tumoren, welche heute fast ausschließlich auf dem Gebiet der medikamentösen Tumortherapie durchgeführt werden, sind häufig an Zulassungsstudien neuer Krebsmedikamente gekoppelt. Direkte Therapiestudien, welche Operation und Chemoradiotherapie bzw. Bioradiotherapie vergleichen, sind aus ethischen und studientechnischen Gründen sehr schwierig und existieren bis heute im Kopf-HalsGebiet kaum. Seit einigen Jahren besteht die Standardtherapie von nichtresektablen Kopf-Hals-Tumoren in einer primären simultanen platinbasierten Chemoradiotherapie. In einer ganzen Reihe aktueller Multizenterstudien ist die Induktionschemotherapie mit TPF (Docetaxel, Cisplatin, 5-Fluorouracil) untersucht worden. Derzeit werden weitere neue Substanzen, v. a. neue Antikörper, als Ergänzung zur Induktionschemotherapie untersucht. Ein Vorteil dieses Therapieansatzes scheint die Selektion von Patienten zu sein, welche von einer medikamentösen Tumortherapie profitie- ren. Bis heute ist jedoch nicht letztendlich geklärt, ob eine Dosisanpassung bei bestimmten Tumorentitäten, wie z.B. HPV-assoziierten Oropharynx- und Mundhöhlenkarzinomen möglich ist. Fragen der Toxizität der nichtoperativen Therapie und die hohe Komplikationsrate der häufig notwendigen Rettungschirurgie werden heftig v. a. von chirurgischer Seite diskutiert. Vor dem Hintergrund der neuen nichtoperativen Therapieansätze muss der Stellenwert der primären chirurgischen Therapie bei KopfHals-Tumoren neu definiert werden. Die Probleme der chemotherapieassoziierten Frühtoxizität und strahlentherapieassoziierten Spättoxizitäten spielen zunehmend eine Rolle bei der Beurteilung der Therapieergebnisse. Mukositis und Knochenmarkdepression, Schluckstörungen, Beeinträchtigungen der Kommunikationsfähigkeit und Nervenausfälle beeinflussen die Lebensqualität. Neben den Überlebenskriterien stellen Organ- und Funktionserhalt nur in der gemeinsamen Betrachtung wesentliche Kriterien für die Beurteilung einer Therapiestrategie bei Kopf-Hals-Tumoren dar. Der zukünftige Stellenwert der primären operativen Therapie mit ihrem besseren funktionellen Ergebnis hängt von der Realisierung prospektiver randomisierter chirurgischer Studien ab. Daneben ist es besonders wichtig, die Möglichkeiten und Nebenwirkungen der medikamentösen Tumortherapie zu kennen.

Mit mehr als 800 Vorträgen, Postern, Referaten, Hauptvorträgen, Rundtischen und Podiumsdiskussionen konnte ein wissenschaftlich anspruchsvolles Programm gestaltet werden. Als Veranstaltungsort der diesjährigen Jahresversammlung wurde das moderne, im Stadtzentrum unmittelbar am Rhein gelegene Congress Centrum Mainz mit der Rheingoldhalle gewählt. Es ist für einen großen Kongress mit seinen vielfältigen Veranstaltungen hervorragend geeignet. Auch die HNO-Akademie hat wieder ihren festen Platz im Ablauf des Kongresses. Die Landeshauptstadt Mainz bietet mit ihren zahlreichen kulturellen und kulinarischen Möglichkeiten einen hervorragenden Rahmen für unsere Jahresversammlung. Ich wünsche allen Teilnehmern einen erfolgreichen Kongress mit vielen Gelegenheiten zum Wissensaustausch und einen angenehmen Aufenthalt in Mainz.

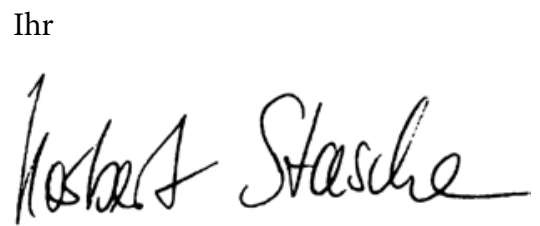

Norbert Stasche

\section{Korrespondenzadresse}

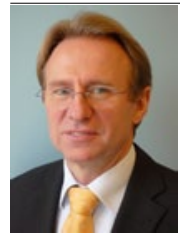

Prof. Dr. N. Stasche

HNO-Klinik

Westpfalz-Klinikum

Kaiserslautern

Hellmut-Hartert-Str. 1,

67655 Kaiserslautern nstasche@westpfalzklinikum.de 